\title{
LA CIENCIA POST NO-CLASICA Y LA FILOSOFÍA
}

\author{
Ricardo BURGUETE AYALA \\ de la Academia de Ciencias de Rusia \\ Prof. invitado UNED
}

Hoy día, cuando nos preparamos a traspasar el humbral del siglo XXI, es evidente, que entre todas las formas de la conciencia social la ciencia es la que goza de mayor prestigio y autoridad y a la que debemos nuestra concepción del mundo, nuestro modo de ser, conocer y actuar.

El siglo XX se puede definir, sin dudas, como el siglo de la Ciencia y ha sido ésta la que, en gran medida ha determinado las peculiaridades básicas de nuestra Civilización y las características del desarrollo de la Cultura. Entendiendo por Civilización una forma histórica específica de relaciones entre el Hombre y la Naturaleza, cuya esencia radica en la forma de asimilación por el Hombre del "cuerpo físico" de ésta y su transformación en objetos artificiales para su uso y consumo, así como por la elaboración de instrumentos, procedimientos y métodos de esa "asimilación" o "apropiación". Todo lo que creado por el Hombre en este proceso ha recibido el nombre de "Segunda naturaleza" o Cultura.

El desarrollo de la Civilización, y si se quiere su progreso, implica un perfeccionamiento constante o una optimización de estas relaciones, pero también un incremento de sus dos tipos básicos de producción: la de objetos y bienes materiales y la producción de ideas, o sea, la producción espiritual, es decir, la creación de la Cultura y sus formas: la Ciencia, la Filosofia, la Religión, el Arte, etc. Las que han recibido el nombre de "formas de la conciencia social".

El papel que juega la ciencia en la sociedad contemporánea no es una simple casualidad, ni una situación pasajera, sino un

Endoxa: Series Filosóficas, $n^{\circ} 4,1994$, UNED, Madrid:

Ricardo Burguete Ayala: La ciencia post-noclásica y la filosofía.

pp. 137-167. 
fenómeno que tiene profundas raíces históricas y refleja ciertas características esenciales de nuestra civilización y nuestra cultura. El tipo de civilización que se gesta en Europa, para el que algunos autores han propuesto el nombre de "Civilización tecnogénica"1, es una civilización dinámica en la que la aplicación de los conocimientos científicos genera no solo una mentalidad renovadora y un estilo de pensamiento propio, sino que tiene como resultado el surgimiento de una gran movilidad en las estructuras y los vínculos sociales, impulsando un proceso continuado de cambios cualitativos en la esfera de la ciencia y la técnica, que se conocen ampliamente con el nombre de "Revoluciones científico técnicas".

Los valores de esta civilización tienen su origen en la antigua Grecia, donde se crean las premisas del pensamiento teórico y surgen los primeros programas científicos o científico-filosóficos. Estos valores se complementan en la Edad media donde, en los marcos de la concepción del mundo religiosa surge, a veces en clara y dramática oposición a la idea de la revelación y al principio de autoridad, una concepción de la razón humana que, al parangonar a esta con la razón divina, se declara capaz de alcanzar y comprender en forma racional el contenido y el sentido del mundo que nos rodeas, los fundamentos del ser y el existir.

En los siglos XVII-XVIII los valores básicos de este tipo de civilización se imponen prioritariamente, abogando por la necesidad de basar el desarrollo social y la solución de sus problemas en

\footnotetext{
1 Ver Stiopin V.S. "El conocimiento científico y los valores de la civilización tecnogénica." Vopr. Fil. N.10. 1989. M.Weber en su libro La ética protestante y el espíritu del capitalismo, fué uno de los primeros que, desde este punto de vista, ofreció un análisis global de las características de la Civilización tecnogénica (sin utilizar este término), al estudiar la génesis de la socidad industrial europea como la manifestación de un tipo específico de racionalidad. Racionalidad que según él se manifiesta en todas las esferas de la cultura desde la actividad económica hasta la vida privada. Fué precisamente Weber quién señaló, que el rasgo más significativo de esta racionalidad era el aumento del papel social de la ciencia.
} 
el aumento gradual y cualitativo de los conocimientos científicos. Desde entonces es la ciencia y el tipo de racionalidad que la caracteriza la que formula los paradigmas básicos que conforman nuestra concepción del mundo y elabora los métodos y principios del saber racional y, en primer término: las formas de demostración y fundamentación de los conocimientos, los métodos de descripción y comunicación de estos, y los procedimientos de organización y sistematización del saber.

La ciencia no solo adquiere una personalidad propia que la diferencia claramente de las demás formas de la conciencia social, incluida la filosofía, sino que "impone" a estas, de una u otra forma, muchos de sus principios y métodos, pero también sin dudas, algunos de sus mitos y prejuicios.

La fe en el valor del conocimiento científico y el poder de la ciencia terminó fomentando una relación equivocada entre el Hombre y la Naturaleza, relación en la que éste se consideró una realidad superior, capaz no solo de descubrir e interpretar los "secretos" de la naturaleza, sino de establecer su dominio sobre ella, transformándola y aprovechándola según sus necesidades objetivas pero, con frecuencia y lamentablemente según su antojo. El conocimiento llegó a considerarse no una forma de diálogo entre el Hombre y la Naturaleza, sino mas bien un instrumento de dominio sobre ésta.

En esta situación, la filosofía ha pretendido con frecuencia ser un saber similar al científico, algo así como una "superciencia" o una metateoría del saber, exigiendo para si un lugar preferencial en "el sistema general de las ciencias". Se ha hablado de una "ciencia de las ciencias", de una "ciencia general" o de la "ciencia sobre las leyes mas generales". Pero además, la filosofía ha seguido reclamando paro si el "privilegio" de la fundamentación teórica de todo tipo de saber y en primer término del científico, quedando de esta manera atrapada en un círculo vicioso, al pretender convertirse en la fundamentación de si misma, o sea, ser ciencia y una fundamentación externa de esta. No obstante, parece evidente, que si la filosofía quiere en forma efectiva cumplir su verdadera 
función de autoconciencia de la cultura o simplemente de autoreflexión del conocimiento, incluido el científico, debe ser necesariamente diferente de este.

Esto significa que la supuesta perfección de los modelos de la relación objeto-sujeto que nos ofrece la ciencia en sus diferentes períodos o etapas de desarrollo no pueden ser aceptados por la filosofía, la que ya desde sus inicios ha elaborado por cuenta propia una concepción del objeto y el sujeto del conocimiento y una tipología de sus relaciones, más perfectas que las que se ha querido imponer a partir de los modelos particulares de la "ciencia clásica" y mas tarde de la "relativista". Lo que, no obstante, no es un obstáculo para valorar altamente el papel positivo realizado por el tipo de racionalidad que caracteriza la ciencia occidental, desde sus etapas iniciales y que adquiere en la sociedad tecnogénica desarrollada un nuevo impulso, debido a los modelos del saber que se imponen en la evolución de la ciencia a partir de los siglos XVII - XVIII, ejerciendo una influencia casi decisiva en los sistemas e ideas de los filósofos.

Desde entonces, en el desarrollo o en la evolución del pensamiento científico se suelen destacar tres periodos que se definen, tanto por su contenido específico y tipo de racionalidad, como por el carácter de las relaciones que se establecen entre la ciencia y los diferentes componentes de la cultura y, en primer término, entre la ciencia y la filosofía.

Al establecer esta periodización se ha partido de la idea de que la propia ciencia, que es un sistema abierto en autodesarrollo, ha pasado por tres estados sistémicos. Cada uno de los cuales tiene una teoría básica que se constituye en paradigma y en los marcos del cual se formulan los ideales y las normas de la cientificidad. La mecánica de Newton primero, luego la teoría de la relatividad y la mecánica cuántica y, en la actualidad la termodinámica de desequilibrio y su más amplia generalización - la sinergética. A cada uno de estos estados corresponde una comprensión propia del objeto de la ciencia y de la relación objeto - sujeto, así como 
una forma específica de autoreflexión del sujeto sobre el propio proceso cognoscitivo, es decir un estilo específico de filosofar. El primero de estos y más largo por su duración es el de la "Ciencia clásica", que se prolonga hasta fines del siglo XIX; el segundo es el de la "Ciencia no clásica" o relativista, que mantiene su vigencia hasta los años $60 \mathrm{del}$ siglo $\mathrm{XX}$ y que posiblemente sea un periodo de transito o la etapa inicial de otro más extenso y cualitativamente distinto del "clásico", cuyos rasgos ya se vislumbran en lo que ahora se ha definido como un tercer período, que corresponde a nuestros días, está en gestación y no ha recibido aun su nombre definitivo, pero se suele denominar "Post-noclásico" o "neo-noclásico" y se basa en una concepción del mundo radicalmente distinta tanto de la mecanicista como de la relativista.

En este momento no tenemos ni la intención ni la posibilidad de analizar detalladamente las implicaciones que en cada uno de estos periodos han caracterizado la interrelación de la filosofía y la ciencia tanto en lo que se refiere a la influencia que sobre el pensamiento filosófico han ejercido lo modelos cognoscitivos y los métodos de la ciencia, como el papel de la filosofía en la fundamentación teórica del pensamiento científico.

Centraremos nuestra atención solo en el último de estos, pero antes quisiéramos señalar, si entrar en detalles, algunas de las características mas relevantes de los periodos precedentes, para que nos sirvan de puntos de referencia en nuestra exposición. Que la filosofía y la ciencia han mantenido durante su larga historia relaciones muy específicas, distintas de las que también han existido entre estas y otras formas de la conciencia social, es un hecho evidente y ampliamente reconocido. A pesar de ello, en cada nueva etapa del desarrollo de la cultura este tema se vuelve a plantear y su análisis y estudio descubre nuevos matices y problemas.

Entre los múltiples factores que en cada uno de estos períodos han influido decisivamente en la mentalidad científica "determinando" el carácter de esta relación e influyendo decisivamente en la tipología de la reflexión filosófica, un lugar muy importante 
pertenece a los ya mencionados principios e ideales investigativos. La existencia y el papel de éstos, se confirma fácilmente con una simple visión panorámica de la historia de la ciencia y la filosofia, permitiéndonos afirmar que entre científicos tan diferentes como Galileo, Newton, Boyle, Lorentz, Descartes, Hertz, Laplace, Lamark, y otros muchos, que representan el primer periodo, es posible establecer una coincidencia en lo que a estos ideales y principios se refiere, y que son estos los que los distingue claramente de científicos como Einstein, Bohr, Bertalanffy, Dirac, Heisenberg, Planck, Wiener...que representan el segundo periodo. Pero también nos ofrece la posibilidad de distinguir a aquellos que, de una $\mathbf{u}$ otra forma rompían con los ideales y modelos tradicionales de su época, como es el caso de Lobachevsky, Darwin, Galois o Mendel.

El surgimiento de la "Ciencia clásica" y sus métodos representó una ruptura con el estilo de pensamiento y los ideales cognoscitivos que se imponen en la Edad media, los que estaban en concordancia con una concepción del mundo en la que la religión $y$ otras formas de aprehensión de la realidad jugaban un importante $\mathrm{y}$ a veces determinante papel.

Cuando a fines del siglo XVII Newton formula las ideas fundamentales de su teoría científica de la Naturaleza surge la falsa esperanza de que por fin se habian logrado superar las dificultades que no habían permitido penetrar en los misterios de la naturaleza, y conseguido formular un sistema integral del saber y creado un método universal para su conocimiento. Ahora ya no existían obstáculos reales para culminar la creación de un verdadero cuadro científico del mundo. $Y$ a pesar de que desde un primer momento existieron diferentes interpretaciones sobre el alcance del sistema y método newtoniano, la idea generalizada y con el tiempo aparentemente confirmada, fue el reconocimiento de su universalidad y perfección. Se consideró que la estrategia investigativa de Newton, al ser aplicada no solo a la naturaleza sino también a la sociedad, nos descubriría en forma univalente e inequívoca las 
leyes objetivas de su existencia y desarrollo y nos daría el dominio sobre la Naturaleza.

Esta estrategia consistiría en el descubrimiento de un hecho o de una realidad fundamental, que luego se utilizaría como base de todas las construcciones teóricas deductivas. la gravitación universal es su mejor ejemplo. Ahora sabemos que se trataba de un cuadro del mundo mecanicista que formulaba un sistema de leyes y principios basadas en la idea de la estabilidad y equilibrio de los sistemas. Pero, durante todo el período que sigue a la "Revolución newtoniana", esta fe en el saber creó el mito que alienta toda la Ciencia clásica: la creencia en la posibilidad de descubrir una verdad radical, la clave de una comprensión universal y única de la naturaleza, que nos permitiría alcanzar un nivel fundamental del ser que haría posible abarcar y comprender todo lo existente. Esta falsa ilusión la señaló acertadamente Einstein cuando dijo "que los postulados generales que constituyen las bases de las construcciones mentales de la física teórica pretenden ser efectivos para todos los sucesos que tienen lugar en la naturaleza".

La concepción de mundo newtoniana se convirtió paulatinamente en la clave tanto de los sistemas científicos como filosóficos de la Época moderna. El sistema newtoniano fue considerado como la base del modelo universal de la ciencia. Se llegó a pensar que el método racional de Newton era la única alternativa para un diálogo productivo con la naturaleza, y la apropiación racional de esta por el hombre. Con la concepción Newtoniana de la naturaleza y su conocimiento culmina la etapa inicial de formación del cuadro científico del mundo, el proceso de formación de la Ciencia. Para la elaboración de su sintesis Newton hizo suyas algunas ideas básicas que inspiraron los programas científicos de la antigüedad clásica y son fundamentales para nuestra civilización y entre estas:

2 Einstein A. Recopilación de trabajos científicos. T.4.Moscú,1967 pág. 40 
- La idea de que el saber racional y objetivo de la ciencia es, ante todo, un modo de actuar sobe el mundo, un conocimiento activo, un medio de acción sistemática sobre la realidad, que permite predecir los posibles resultados de la acción cognoscitiva y utilizar mejor, con la creación de instrumentos y artefactos, las fuerzas y los recursos de la naturaleza.

- El afán por comprender racionalmente el mundo y el lugar del hombre en él. Esta búsqueda se caracterizó, a diferencia de la aprehensión religiosa, por su espíritu crítico por cierta "obsesión" de encontrar para todos los fenómenos naturales una explicación racional y un método eficaz y seguro de verificar los resultados obtenidos.

- La "Revolución newtoniana" ofreció una solución al problema que había preocupado seriamente a los creadores de los primeros programas científicos: cómo superar las diferencias que parecían existir entre el Universo aparentemente "apacible y tranquilo", sometido a la acción inexorable de las leyes eternas e inmutables, y el mundo de los hombres, siempre conflictivo, cambiante e impredecible.

El estilo de pensamiento que se impone en nuestra cultura a partir de la "Revolución newtoniana" y que caracteriza la mentalidad científica e intelectual de este período tuvo una resonancia especial en las ideas y los sistemas de los filósofos, influyendo tanto en la concepción del mundo como en la "epistemología" filosófica y sus problemas.

Entre los temas que preocuparon y dominaron este nuevo estilo de pensamiento $y$, en cierta manera, determinaron los rumbos de la reflexión filosófica, así como la relación entre la filosofía y la ciencia, quisiéramos destacar dos. El período durante el cual surgen las ciencias naturales modernas y cuyos marcos temporales van del Renacimiento hasta fines del siglo XIX, se caracteriza por un tipo de pensamiento que a veces se ha definido como "Racionalismo clásico". Este racionalismo "determinó" no solo los rasgos mas relevantes del saber científico sino también las normas e ideales del conocimiento en general. Su fundamento fue la profunda 
convicción de que la razón cognoscente debe procurar diferenciarse y distanciarse del objeto conocido. La aprehensión cientifica si de verdad quiere ser tal, debe lograr una visión externa de su objeto. Esto significa que el ideal de la descripción teórica, la objetividad, exigía la eliminación de todo lo relacionado con el papel del sujeto y, en primer término, la exclusión de la influencia que sobre el objeto hayan podido ejercer los métodos, las operaciones y los medios empleados para su conocimiento. La fuerza de este ideal causó serias confusiones entre no pocos filósofos, que también buscaron la objetividad y la veracidad de sus doctrinas en una separación radical entre el objeto y el sujeto de la reflexión filosófica, "olvidándose", por así decirlo, que el objeto de la filosofía no era una realidad externa diferente del sujeto sino, en gran medida, el propio sujeto asi como las relaciones objeto sujeto en el proceso del conocimiento y actividad práctica del hombre.

El otro aspecto, que nos ha parecido importante señalar, está estrechamente vinculado a esta interpretación "cientificista" del objeto y el sujeto del conocimiento. Nos referimos al Método experimental, que algunos autores consideran uno de los logros más importantes de nuestra cultura, definiéndolo como un "diálogo experimental del hombre con la naturaleza". 3

Según este criterio, la complejidad de la naturaleza es solo una apariencia y su diversidad se puede reducir a ciertas leyes universales simples y comprensibles (para Galileo las leyes matemáticas del movimiento).

Se puede decir que para su tiempo esta idea fue, en gran medida, productiva y útil. Colaboró eficazmente en la lucha contra ciertas tradiciones metafísicos y teológicas y estimuló la aplicación de los métodos experimentales. No obstante quisiera destacar que la afirmación de Prigogin de que, en cierto momento este mecanicismo encontró su punto de contacto no solo con la metafísica

\footnotetext{
3 Ver I.Prigogine y M.Stengers Order out of chaos (Mans new dialoge with nature) Heinemann. London. 1984, págs 84 - 88.
} 
escolástica sino con el pensamiento teológico, nos parece acertada. Prigogin dice que entre ellos se establece un determinado tipo de "resonancia" de la que todos salen favorecidos.

Esta situación está estrechamente vinculada a otra de las características mas significativas de la etapa inicial de la ciencia clásica, que en cierta manera se ha manifestado durante todo el período de su existencia: la posibilidad real de la labor simultánea del científico y el teólogo. Esto explica el hecho bien conocido, de que durante todo este período teólogos y filósofos pudieran dedicarse con mayor o menor éxito a investigar los fenómenos naturales y los científicos a descifrar la sabiduría divina y los propósitos ocultos del Creador.

Precisamente en los marcos de esta "resonancia" la física y la teología se enfrentaron, en los inicios de la Época moderna, al programa científico de Aristóteles, cuyo "mundo" resultó inaceptable para unos y otros, ya que era un "mundo" demasiado heterogéneo y complicado para ser expresado por las leyes y las fórmulas matemáticas. Con frecuencia esta "resonancia" se presenta en un mismo pensador, lo que se descubre fácilmente con la simple pero atenta lectura de sus obras y no exige un esfuerzo investigativo especial. Una lista tentatoria con los nombres de famosos científicos y filósofos de este largo período, desde su gestación en el siglo XV hasta el XIX mostraría salvo raras excepciones, que en cada uno de ellos se puede encontrar un vertiente teológica un esfuerzo por dar una respuesta positiva a la pregunta sobre el lugar de Dios en ese mundo sometido a las leyes de la mecánica, que nos descubre y describe la ciencia. $Y$ esa coincidencia tiene lugar a pesar de sus múltiples diferencias tanto en sus "programas científicos" como a las ideas religiosas.

Esta situación, o este hecho, tiene un profundo contenido teóricometodológico y descubre, aunque después de lo dicho parezca paradójico, una de las facetas más importantes que caracterizan el paradigma científico de este período: la expulsión de la ciencia de la idea de finalidad. Preguntar a la Naturaleza sobre sus fines es una prerrogativa de la "Metafísica" y de la Teología. Esto lo 
declaran explícita y tajantemente pensadores tan diferentes como Descartes, Bacon, Spinosa, etc. tradición que continua la Ilustración, y resume magistralmente Kant al mostrar que la categoría de "finalidad" no pertenece a la razón teórica y tiene valor solo para la filosofía práctica.

La revolución en las ciencias naturales, que tiene lugar a fines del siglo XIX principios del XX, y cuya expresión mas evidente es la Física relativista cuántica, crea un nuevo tipo de racionalidad que marca el inicio de la transformación de los ideales y normas del conocimiento científico de la época clásica.

La esencia de esta nueva racionalidad tiene como núcleo central el reconocimiento de que el sujeto no está separado, ni se puede separar en forma radical, del objeto de la investigación, sino que está dentro de este. Es decir, el investigador se encuentra o se debe colocar dentro de la realidad investigada, y el conocimiento se realiza gracias a la actividad del sujeto en ella, cosa que la filosofía había afirmado hace mucho tiempo al decir que, solo cuando los objetos resultan incorporados a la actividad humana surge la posibilidad real de conocer sus vínculos esenciales. Esto significa la aceptación evidente de que la descripción teórica realizada por la ciencia no puede ya ignorar ni el carácter ni las peculiaridades ni la finalidad de nuestra actividad investigativa, y siempre debe tener presente el "lugar" desde el que el investigador formula sus preguntas a la realidad.

Durante este período las ideas fundamentales de la ciencia clásica fueron socavadas por una serie de factores que coinciden en sus críticas a la concepción del mundo mecánico-determinista. Tiene lugar un cambio importante en la mentalidad intelectual, que se refleja en la estructura de la producción espiritual, dando paso a un nuevo tipo de racionalidad, que se ve obligada a admitir que la conciencia al aprehender la realidad no puede ignorar el hecho de que se encuentra sumergida en esta, es parte de ella $y$, ademas, depende del medio social que condiciona la finalidad y la valoración del conocimiento. 
Ya a principios del siglo $X X$ se elaboran teorías e hipótesis científicas que rompen definitivamente los esquemas básicos de la física clásica. La teoría probabilística del electrón de M.Boorn, la Mecánica ondulatoria de L.de Broglie, las teorías de la Relatividad de A.Einstein, la Física cuántica de M.Planck etc. crean el "desconcierto", planteando la necesidad urgente de una revisión seria de los postulados y principios de la ciencia clásica. Se hace evidente que los nuevos descubrimientos ya no encuentran en ésta una interpretación teórica satisfactoria y que la descripción determinista es ya inoperante para ello. Por eso N.Bohr tiene que reconocer que:

"Si por una parte la descripción que ofrece nuestra actividad intelectual nos exige la contraposición entre el contenido objetivo de la realidad y el sujeto pensante, por otra, como es evidente, no es posible diferenciar rigurosamente el objeto del sujeto, ya que este último también es parte del contenido."

A lo que pudiera añadirse esta observación de Pauli, que se ha confirmado plenamente en la ciencia contemporánea:

"Yo hasta supongo que el observador en la física contemporánea está aún demasiado aislado, por lo que la física cada vez se apartará más de los modelos clásicos. ${ }^{15}$.

Por su parte I.Prigogin al señalar los motivos de esta crisis añade que esto se ha hecho evidente cuando en la física en calidad de objetos del saber positivo se han presentado la casualidad, la complejidad y la irreversibilidad:

"Ahora las descripciones que nos proporciona la ciencia, ya no pueden ser separadas de nuestra actividad investigativa." 6

Por todo esto, parece evidente, que no es una exageración la afirmación de que una de las características mas relevantes en la evolución de la ciencia contemporánea es la participación, cada vez

\footnotetext{
4 N.Bohr Obras escogidas. T.2. pág. 58. Moscú 1971.

Pauli. Ensayos de Física. págs. 26-27. Moscú 1975

6 I.Prigogine, I.Stengers Order out of chaos. London 1984. pág101 de la Ed. rusa Moscú, 1986.
} 
más amplia, del sujeto en la estructura del saber positivo, lo que crea una situación nueva para el acercamiento del saber científico y el filosófico. Esta situación de "incertidumbre" o de crisis se expone con exactitud trágica en la Introducción que nos ofrece F.Suppe al libro La estructura de las teorías científicas, en el que se refleja el estado del problema a fines de los años 60 .

Suppe coloca en el centro de su exposición la crítica a que fue sometida la llamada "Concepción heredada", pudiera decirse, la filosofía y la metodología de la ciencia de la época clásica, de ofrecer una respuesta adecuada y bien fundamentada a los recientes descubrimientos de la física, debido a su manera de concebir las teorias y el conocimiento científico en general. El resultado de esta crítica, dice:

"...fue, que la concepción heredada resultó destronada, pero ninguna de las alternativas propuestas ha ganado aceptación general entre los filósofos de la ciencia. La filosofía de la ciencia cae asi en un estado de aguda confusión intelectual". A pesar de que "Durante más de cincuenta años la filosofía de la ciencia se ha dedicado a la búsqueda de la comprensión filosófica de las teorias científicas; hoy sigue todavia buscando."7

Es evidente, y así lo muestra explícitamente el libro de Suppe, que la crisis de la Concepción heredada refleja entre otras cosas la incapacidad de resolver el problema de la fundamentación filosófica de la ciencia, y entre otras cosas, el fracaso en esta empresa de la filosofía positivista. Pero esta crisis va mucho mas lejos de los sistemas filosóficos analizados en el libro, entre los que se debería incluir el marxismo, tanto de los "Clásicos" como el "Dogmático", que se quiso imponer oficialmente en nuestro siglo; pero también todas aquellas doctrinas filosóficas que al declarar que la filosofía es también una ciencia ha quedado atrapadas por el "Paradigma gnoseológico" de la ciencia clásica, cuyo modelo han seguido.

Federico Suppe. La estructura de las teorías científicas. UNED. Madrid,1990. pág. 17. 
El hecho de que, actualmente ningún análisis de las teorias goce de aceptación general entre la mayoría de los filósofos de la ciencia, hace pensar, que posiblemente la solución al problema se ha seguido buscando en un lugar poco apropiado para ello, es decir, en el análisis interno y formal de las teorías. Aceptando como un "postulado" irrevocable la afirmación que:

"El problema central de la filosofía de la ciencia es el de la naturaleza o estructura de las teorias cientificas. ${ }^{8}$

Postulado que, al parecer, debería ser perfeccionado o seriamente revisado, buscando la solución del tema desde una perspectiva más amplia Pero para que esta revisión sea posible, habrá que reconocer que hasta ahora no se han logrado superar las limitaciones implícitas en el modelo del pensamiento teórico de la Ciencia clásica ni los prejuicios de la concepción del mundo mecanicista, que han dominado el pensamiento científico y filosófico durante toda la época moderna de nuestra Cultura, desde Galileo y Newton hasta nuestros días.

Desde que Suppe escribió su libro ya han pasado algunos años, pero hoy se puede decir que entre los historiadores de la ciencia, incluidos los filósofos de la ciencia, no se ha logrado aún suficiente claridad y consenso sobre los parámetros y tipología de los cambios que caracterizan el desarrollo del pensamiento científico, es decir, de la Ciencia. Se llamen estos "Revoluciones científicas", "Estilos de pensamiento", etc., no hay acuerdo sobre los criterios y principios a los que se debe recurrir para su periodización. En este sentido estamos de acuerdo con Suppe cuando dice que esto se debe a que:

"Han hecho de la ciencia algo demasiado subjetivo, demasiado misterioso y demasiado ligado a construcciones metafisicas; y sus análisis requieren excesivas referencias a factores sicológicos $y$ sociológicos. Lo que se precisa es un análisis filosófico de la actividad

8 Op. cit. pág. 15. 
del teorizar científico que no necesite recurrir ni a factores sicológicos ni sociológicos ni a sus reificaciones como weltanschauungen."

Entre los muchos intentos de abordar la solución de este tema, la teoría "paradigmal" de T.Kuhn ha sido, posiblemente una de las más conocidas y discutidas. Inicialmente se consideró muy prometedora, pues parecía explicar el desarrollo de la ciencia hasta el siglo XX. Pero a la hora de abordar los problemas y los cambios que tienen lugar en la física contemporánea resultó poco efectiva desviando su atención, como reconoce el propio Kuhn, hacia el estudio de las colectividades de científicos y las incidencias de sus características sociológicas y psicológicas en el proceso del conocimiento, aportando muy poco para el análisis de la ciencia post-relativista, cuyo nacimiento y significado parece no haber advertido. Hoy día, es necesaria, ante todo, una visión clara de aquellos elementos del paradigma de la ciencia clásica que han resultado superados por el desarrollo de las ciencias contemporáneas y deben ser revisados. He aquí los más importantes:

- el caos es un factor destructivo y por lo tanto negativo;

- la casualidad, la contingencia es irrelevante para la Naturaleza, la ciencia y la Cultura;

- el mundo y su desarrollo no dependen ni de las microfluctuaciones ni de los microprocesos cósmicos;

- el desequilibrio y la inestabilidad son factores negativos incómodos y desagradables, que deben ser superados por alterar el curso "correcto" de los sucesos;

- el desarrollo transcurre en forma lineal, sin alternativas. (la variante dialéctica del desarrollo en "espiral" no es una excepción).

- Las posibles alternativas, no son nada más que desviaciones temporales del curso correcto de los sucesos, determinado, en última instancia, por las leyes objetivas universales del devenir;

- todas las alternativas están sometidas a la acción niveladora de las leyes universales;

9

Op. cit. págs. 351-352. 
- el desarrollo es predecible en ambas direcciones, ya que las leyes universales son atemporales;

- los procesos y sistemas tanto naturales como universales se pueden gobernar a partir de una concepción lineal determinista del desarrollo de la naturaleza y la sociedad;

- toda acción rectora u organizativa está en proporción directa con el esfuerzo empleado según la formula "más energía mejor resultado". Esta falsa idea ha sido una de las causas del desastre ecológico actual;

- y por último cabe señalar, que el estilo de pensamiento de la ciencia clásica (que se ha definido como lineal) considera los diferentes procesos que estudia como si fueran procesos cerrados en los que todo transcurre según el segundo principio de la termodinámica, lo que significa que van hacia la degradación, hacia una situación de máxima entropía y hacia el caos.

El "dramatismo" de la situación actual de la ciencia, ante el cambio de paradigma, se agudiza por el hecho de que por diversas causas la filosofía también está pasando por una situación crítica, en la que parece haber perdido, o no tener muy claro, su lugar y su función específica en el sistema de la cultura y el papel que debe jugar en este momento en la fundamentación del conocimiento en general y el científico en particular.

Como hemos señalado, uno de los motivos de ello ha sido el empeño de algunos filósofos en convertir la filosofía en una ciencia, haciendo de la teoría filosófica del conocimiento un "apéndice" del método experimental, y del racionalismo filosófico en una técnica para la conquista y la transformación de la realidad. ${ }^{10}$

\footnotetext{
10 Tarea planteada explicitamente por C.Marx a la filosofía en una de sus tesis sobre Feuerbach donde afirma "Los filósofos solo han explicado el mundo de diferente forma, pero la tarea consiste en transformarlo". Ver C. Marx y F. Engels Obras. T. 3 págs 1-5
} 
Es evidente, que hoy se necesita un cambio de racionalidad, que tenga como meta la búsqueda del verdadero sentido, no solo de los actos humanos y los hechos sociales sino de los fenómenos de la naturaleza, que van más allá de las simples relaciones deterministas de causa y efecto y siguen unos ritmos y lineas de desarrollo que exigen para su comprensión una idea de la finalidad más amplia y generosa que la utilizada hasta ahora, la que ha expulsado de la Naturaleza no solo el tiempo y la irreversibilidad sino también el sentido.

Y si este cambio se está produciendo, esto se debe, en gran parte, al esfuerzo que están realizando las ciencias físicas en el estudio de los procesos y fenómenos que tienen lugar en los sistemas complejos autoorganizados. Este estudio se ha convertido en un factor decisivo en la consagración de un paradigma que, al hacer su aparición a mediados del siglo pasado en la teoría biológica de la evolución, parecía confirmar la existencia real de dos mundos o dos órdenes diferentes, regidos por leyes y regularidades cualitativamente distintas : el físico que, según las leyes de la entropia, marchaba hacia la simplicidad y el biológico que, violando estas leyes, se movía hacia una mayor organización y complejidad. Merece la pena señalar que ya entonces, en el seno de la propia física, se valoró positivamente la perspectiva metodológica que para la ciencia en general ofrecía la teoría de la evolución. Boltzmann, por ejemplo, llegó a afirmar que el siglo XIX era el siglo de Darwin.

Es cierto, que ya en el siglo XIX en la propia física, pero aún en forma incipiente, las ideas de este nuevo paradigma se pueden vislumbrar en los trabajos de Boltzmann y Puancare, en los que se habla de la física de los sistemas de disipación, de los atractores etc. $y$ en los que se anuncian los posibles caminos de una nueva física. 
"La convicción de Boltzmann de que la irreversibilidad constituye la médula de la física hoy está confirmada: la irreversibilidad ha abierto para la física el problema del devenir."

El hecho de que la física sea una fuente o una impulsora de nuevos paradigmas globales, pudiera decirse, de una nueva concepción del mundo no nos debe sorprender, pues lo ha sido en otros casos. El paradigma determinista es uno de ellos, el relativista y probabilístico otro.

El actual se basa en los conceptos de inestabilidad, desequilibrio, no linealidad, irreversibilidad del tiempo, etc. Como todo nuevo paradigma y como toda nueva concepción del mundo en formación, aún debe ser sometido a una reflexión filosófica, a un estudio que determine lo que las nuevas ideas implican para todo el sistema del saber contemporáneo y, en primer término, para la propia filosofía y sus relaciones con la ciencia.

Pero a pesar de que efectivamente, los procesos en los sistemas físicos, químicos o biológicos se pueden dividir en dos grupos: los que tienen lugar en sistemas relativamente cerrados y en su desarrollo tienden hacia una situación de equilibrio y máximo desorden en el movimiento de sus componentes, es decir, hacia el caos físico, cuya medida es la entropía y los que, a partir de ese caos, surgen en la evolución temporal de los sistemas abiertos como resultado de la autoorganización dando lugar a nuevas estructuras, las llamadas "estructuras de disipación"; es no menos evidente que existe la posibilidad y, más aún, la necesidad urgente, de elaborar una teoría única que logre explicar los dos tipos de procesos.

No cabe duda, que el nuevo estilo de pensamiento está cambiando radicalmente nuestras ideas y el modo de enfocar los problemas en todas las esferas de la actividad teórica y práctica. Los modelos de equilibrio se sustituyen por los de desequilibrio y ahora el mundo físico en todos sus niveles, como antes el biológi-

\footnotetext{
11 I.Prigogin "El redescubrimiento del tiempo". Voprosy Filosofii, N. 8,Moscú, 1989 pág. 13.
} 
co, es verdaderamente un mundo dinámico y temporal, y lo que las "leyes universales" del paradigma determinista "negaban" o "censuraban" ocupa ya un lugar preferencial en el nuevo cuadro del mundo que nos ofrece la ciencia.

Respondiendo a la necesidad de esta sustitución, en la segunda mitad del siglo XX surge una dirección interdisciplinaria de la ciencia que tiene como objeto el estudio de las regularidades y las características de la evolución y organización del mundo no lineal, y en cuyos marcos nace la "Sinergética", que se define como la ciencia que estudia los sistemas no-lineales de autoorganización. Se trata de una ciencia "post-noclásica" cuya afinidad con la filosofía y sus problemas es mucho mas estrecha que la existente entre la ciencia y la filosofía en épocas anteriores.

Aún no se ha escrito la historia del nacimiento de esta nueva ciencia, pero quien lo intente deberá tener presente estas reveladoras palabras:

"Los primeros 'sinergéticos' fueron Kant y Laplace, que construyeron a partir de la nebulosa caótica primaria el modelo teórico del surgimiento del sistema solar. El segundo fue Darwin, que a partir de la variabilidad casual caótica descubrió el mecanismo del surgimiento y desarrollo de la biosfera ordenada. Este mecanismo fue la selección natural. El modelo de Kant y Laplace hoy tiene solo interés histórico. Por el contrario la teoria de Darwin, es uno de los mayores logros del pensamiento humano y conserva plenamente su significado. Darwin es el fundador de la "Sinergética" a pesar de que sobre física escribió menos que sobre el origen de las lenguas."12

Como hemos señalado, el ideal de la ciencia "clásica" pretendia que sus descripciones teóricas reflejasen en forma adecuada la realidad, de tal modo, que cada concepto científico tuviese un correlato en el mundo objetivo, es decir un representante ontológico indiferente a la acción del sujeto y a los medios y métodos implicados en la investigación.

\footnotetext{
12 M.Volkenstein. "La física contemporanea y la biología." Voprosy Filosofii. N. 8. Moscú, 1989, pág. 30.
} 
Esta actitud, que lamentablemente aún no ha sido superada en forma consecuente, fue criticada en su tiempo por la filosofía, que señalo acertadamente, que la relación objeto sujeto era mucho más compleja que la sostenida por los científicos. Pero el camino seguido entonces por la filosofía para solucionar el problema siguió rumbos equivocados. Un ejemplo de ello fue el pensamiento neokantiano, que buscó la solución en la minimización de la relación de correspondencia entre las construcciones teóricas y los objetos reales que estás representan, llegando a convertir la teoría en un mero recurso para reflejar en forma aproximada o útil el mundo que, según ellos afirmaban, era siempre una "cosa en si". Las soluciones positivistas y neopositivistas no fueron mas acertadas o afortunadas en esta empresa, mientras que la Concepción Heredada consideró que la solución del problema radicaba en encontrar el modo de impedir la intromisión de la filosofía en los problemas de la ciencia. Llegando a afirmar, como señala Suppe que:

"...los términos teóricos solo se aceptan si se los puede dotar de reglas de correspondencia que den de ellos una definición fenoménica explícita y, por tanto, las molestas entidades metafísicas no pueden ser introducidas en las teorias cientificas". ${ }^{13}$

La ciencia "no-clasica" intentó superar este divorcio, la Teoría de la relatividad es un ejemplo de ello. Pero no fue consecuente en sus conclusiones. Solo la ciencia "post-noclásica" ha planteado, que la única forma de superar el presunto divorcio entre la teoría y la realidad, consiste en colocar legítimamente al investigador dentro de esa realidad, como una parte de esta, para pensar y elaborar la teoría desde dentro. Solo en este contexto tendría la posibilidad de comprender el valor y el significado de los conceptos básicos que caracterizan la ciencia de nuestro tiempo: el caos la inestabilidad, la autoorganización, la casualidad, la irreversibilidad del tiempo, etc. 
La ciencia "post no-clásica" está aportando una visión sustancialmente nueva de la dinámica de los procesos de autoorganización y la evolución de los sistemas naturales, sociales y cognoscitivos. Ofreciendo a su vez una visión diferente del papel constructivo y creador del caos, de la inestabilidad y la casualidad y poniendo fin al mito de que la acción individual de los sujetos históricos es mínima e insignificante y no tiene un valor esencial en el devenir de los macroprocesos.

La esencia de este mito o prejuicio de la "Razón clásica", o sea de la ciencia, se basaba en la convicción, que ya se ha señalado, de que el desarrollo es, en última instancia, un proceso sin alternativas ni fluctuaciones casuales, en el que el conocimiento podía lograr en forma univalente una visión relativamente fiel del pasado e incluso del futuro.

Posiblemente hoy pueda parecer prematuro intentar una síntesis o una enumeración de los problemas que esta nueva visión del mundo está planteando urgente y dramáticamente a la filosofía. Esto parece aún mas difícil, si tenemos presentes las distintas interpretaciones que se han dado a los datos aportados por la ciencia y a las nuevas teorías que los interpretan. Prigogin en su artículo "El redescubrimiento del tiempo" dice, refiriéndose a esta situación, que:

"Aún estamos muy lejos de comprender 'los principios' de los procesos físicos y químicos, que nos dejan un amplio campo para los descubrimientos. La irreversibilidad y la contingencia ya no son para los fisicos una casualidad que puede ser superada por las leyes de la física. Sino que reflejan caracteristicas esenciales del mundo, y nosotros solo empezamos a comprender las preguntas que le hemos formulado. "14

Por todo lo dicho, quisiéramos tan solo destacar algunos de los temas característicos del pensamiento científico "post-noclásico" cuyo alcance filosófico nos parece indiscutible:

Ante todo, se debe señalar, que el nuevo estilo de pensamiento nolineal se basa en una actitud metodológica que exige una mayor 
apertura y receptividad hacia el surgimiento de lo nuevo y una disponibilidad a aceptar que el aumento repentino de pequeñas e "inesperadas fluctuaciones en la macroestructura de los sistemas puede motivar un crecimiento repentino en avalancha, cuyas consecuencias son impredecibles "a-priori", y que no necesariamente constituyen un adelanto, un "progreso" respecto a la situación precedente, pero tampoco representan un retorno a ella. Por lo tanto, el pensamiento no-lineal es incompatible con la idea del cambio como un proceso gradual o dialéctico de desarrollo o involución de carácter cumulativo. Por otra parte, reconoce también que el desarrollo implica tanto un proceso de surgimiento de tendencias divergentes como la fusión o convergencia de las tendencias existentes.

Hoy parece evidente que el caos es un factor que puede actuar como un principio constructivo en el surgimiento y autodesarrollo de los sistemas, siendo precisamente en el caos donde la inestabilidad y las pequeñas fluctuaciones casuales pueden dar origen a nuevos procesos y a nuevos sistemas, los que en su autodesarrollo tienen ante si un amplio abanico de posibles caminos alternativos. Otro de los elementos de la ciencia "post-noclásica" y posiblemente uno de los mas discutidos es el del papel que juega la inestabilidad en el desarrollo de los sistemas. Prigogin, por ejemplo, considera que en la nueva paradigma científica el concepto de inestabilidad sustituye al de determinismo.

"En el mundo determinista - escribe en uno de sus trabajos - la naturaleza puede ser controlada, es un objeto inerte, sometido a nuestros actos de voluntad. Pero si la naturaleza contiene la inestabilidad, como un elemento esencial, entonces tenemos que respetarla, debido a que no podemos predecir lo que puede ocurrir. Hoy la ciencia no es ni materialista, ni reduccionista, ni determinista" ${ }^{\prime 15}$

Se debe señalar que con este punto de vista, ya bastante generalizado, no están de acuerdo aquellos científicos y filósofos que consideran que la inestabilidad y el determinismo no se deben

15

Prigogine I. The Philosophy of Instability. Futures. August. 1989. pág. 397. 
tratar como conceptos incompatibles, ya que por su contenido reflejan diferentes momentos o etapas del proceso de desarrollo. No obstante, todos parecen estar de acuerdo en que la inestabilidad es la condición necesaria del cambio, pues solo los sistemas en estado de inestabilidad son capaces de autoorganizarse y autodesarrollarse, mientras que la estabilidad y el equilibrio son callejones sin salida en la evolución de los sistemas.

Parece ser, que también existe un consenso en el reconocimiento de que la inestabilidad no tiene un carácter absoluto, se manifiesta y existe temporalmente en determinados marcos y parámetros y caracteriza cierto estado de los sistemas, lo que no excluye la estabilidad relativa como un estado transitorio. Tales son todas la macroestructuras de la naturaleza, las formas biológicas, el cuerpo humano, su cerebro, etc. Durante largos periodos su existencia parece, o se puede considerar estacionaria. Los cambios son imperceptibles y no alteran la esencia del sistema, pero la inestabilidad puede surgir o ser provocada en cualquier momento, originando un cambio radical, un desarrollo en avalancha. Esta posibilidad se comenta asi en la revista Voprosy Filosofii:

"De esta manera llegamos a un resultado sorprendente. A pesar de que todo en el mundo es, en general estable, lo es solo $y$, en cierto grado, en forma relativa y durante una etapa que puede ser relativamente larga. Pero los sistemas complejos al alcanzar su máximo desarrollo tienden a disgregarse. La estabilidad nace de la inestabilidad, como resultado de la inestabilidad, esto se debe a que el nacimiento de una formación estructural nueva está vinculada a la casualidad, al caos, a la inestabilidad. Toda estabilidad, tarde o temprano se convierte a fin de cuentas en inestabilidad"16

Merece la pena señalar, que la concepción radical de la inestabilidad es considerada por muchos científicos como un extremismo injustificado, que absolutiza el hecho evidente de que en el momento de la bifurcación la casualidad juega, efectivamen-

\footnotetext{
16 E.N.Kniasev,S.P.Kurdiumov. La sinergetica como un nueva concepción del mundo: un diálogo con I.Prigogin. Voprosy Filosofii. N. 12. Moscú, págs.16-17.
} 
te, un papel decisivo en el desarrollo de los sistemas, lo que no significa que la posibilidad de diferentes variantes evolutivas sea ilimitada. Se ha establecido que, según muestra la experiencia, existe un número finito de éstas, que se puede predecir matemáticamente. Pero es importante señalar que debido a los aportes de la "Sinergética", ahora existe la posibilidad de pronosticar las distintas y probables lineas de desarrollo de los sistemas complejos.

Todo lo dicho permite afirmar con cierta seguridad que estamos ante un nuevo tipo de "determinismo", en el que se presentan prometedoras oportunidades para la participación activa del hombre en un diálogo productivo con la naturaleza. En nuestra mentalidad ha tenido lugar un cambio radical respecto al modo de abordar los problemas relacionados con la dirección de los sistemas autorregulados y en primer término con la naturaleza. Ahora es evidente que el hombre no puede ni debe intentar imponer a los sistemas complejos una pauta de conducta contraria o diferente a la que marca la linea de su propio autodesarrollo. Esto significa, que para interactuar con un sistema sin generar efectos negativos y contraproducentes es imprescindible conocer las formas de coexistencia en él no solo del Hombre y la Naturaleza, sino también los efectos de todo lo creado por éste en su actividad práctica y teórica.

Hoy día la "Sinergética" está aportando valiosas ideas sobre el modo de operar y "dirigir" los sistemas complejos y ha formulado los principios operativos de la formación o "montaje" de tales sistemas, destacando la importancia del efecto sinérgico en la creación de estos.

Con el surgimiento de la "Sinergética", las ideas y el enfoque sistémico, que desde mediados de siglo habían colaborado seriamente a romper los viejos esquemas de la ciencia, por fin se convierten en uno de los componentes básicos de la nueva concepción del mundo. La buscada "Teoría General de los Sistemas" tiene ahora la posibilidad de una interpretación consecuente 
de toda la realidad sistémica, al incorporar a esta los procesos de autodesarrollo.

Otro de los componentes más importantes de la concepción del mundo que nos ofrece la ciencia "Post-noclásica" es, como se ha señalado, la no- linealidad con la que se expresa el hecho evidente de la existencia de una pluralidad de posibles lineas de desarrollo, tanto de los procesos naturales como sociales.

El concepto de lo no-lineal se formó en las matemáticas y con él se expresaba o definía la existencia de un determinado tipo de ecuaciones que ofrecian soluciones "cualitativamente" distintas. Inicialmente se consideró que se trataba solo de posibilidades matemáticas abstractas, sin ningún contenido "físico", pero mas tarde se descubrió que esto ocurría cuando en el entorno de un proceso o sistema, descrito por este tipo de ecuaciones, cambiaba una de las constantes, lo que se reflejaba en el cambio de uno de los parámetros de la ecuación. En determinado momento, cuando el cambio alcanza una situación crítica, el sistema pierde su estabilidad o equilibrio y en el tiene lugar una "bifurcación", es decir, surgen, como mínimo, dos posibilidades o dos caminos diferentes del desarrollo del sistema. Según Prigogin la pluralidad de soluciones de las ecuaciones no-lineales corresponde a la pluralidad de caminos evolutivos de los objetos que estas ecuaciones describen. Pero además esto significa, que estas ecuaciones nos describen el hecho de que en los sistemas abiertos, y estos son todos, el cerrado es una abstracción teórica, la estabilidad se mantiene dentro de ciertos parámetros de su entorno. Pero si en un determinado momento esos parámetros sobrepasan una magnitud crítica, el desarrollo del sistema cambia cualitativamente, ya que en el nuevo entorno se crea la posibilidad del surgimiento de un sistema nuevo.

Son muchos los elementos que integran la nueva concepción del mundo de la ciencia "post-noclásica", pero entre estos el problema de la irreversibilidad del tiempo, es decir, el de su existencia real, es uno de los más importantes, tanto para la ciencia como para la filosofía. 
En el plano ontológico la solución de este problema afecta todos los ámbitos del ser y, en primer término, el significado y el alcance de las leyes formuladas por las diversas ciencias tanto naturales como sociales. Por lo tanto, no sería una exageración afirmar, que se trata del punto de coincidencia de todos los nuevos problemas planteados por el desarrollo de la ciencia contemporánea.

Por fin se reconoce, que no solo la sociedad y los seres vivos tienen historia, sino que esta existe también en el mundo físico y químico. Pero para que exista historia es necesario que se cumplan tres condiciones mínimas: la irreversibilidad, la probabilidad y la posibilidad del surgimiento de nuevos vínculos. Tener historia significa algo más que tener un pasado y moverse "automáticamente" hacia el inevitable futuro. Ante todo quiere decir, tener la posibilidad de participar activamente en la "elección" de ese futuro, que no es una mera repetición de situaciones pasadas. El verdadero significado de esta idea lo expresa muy bien este razonamiento de Prigogin:

"La expansión del calor tiene su fundamento en la diferencia de temperaturas que con ello disminuye constantemente. No obstante para algunos físicos, $y$ en particular para Planck y Boltzmann, lo esencial consistía en que al realizarse el segundo principio, la naturaleza abandonaba la indiferencia, a la que al parecer la inducia la dinámica. Como decía Plankc, el segundo principio era la expresión de que la naturaleza no era indiferente, que tenia "inclinaciones", "preferencias" respecto ciertos estados. Estos estados preferenciales son los que en la física de los sistemas disipativos han recibido el nombre de "atractores". ${ }^{17}$

Con la formulación en el siglo XIX de la Segunda ley de la termodinámica, que anuncia la inevitabilidad de la muerte térmica del Universo, ante la ciencia se plantea la necesidad de analizar las profundas contradicciones derivadas de la exclusión de la temporalidad de los sistemas físicos y químicos y afrontar el reto que la

17 I. Prigogin. " El redescubrimiento del tiempo". Voprosy Filosofii, N. 8 1989. Moscú, pág. 8. 
Teoría de la evolución planteaba a todas las ciencias naturales. Entonces el reto no se aceptó y se pensó que no correspondía a la ciencia opinar sobre este tema, que según el criterio generalizado, era, ante todo, un tema de la filosofía, y de ninguna manera de las ciencias naturales.

Pero los primeros intentos de incorporar el tiempo a las teorías de la ciencia, sin renunciar a los esquemas tradicionales de los períodos anteriores, condujo a un callejón sin salida. Por eso estamos de acuerdo con Prigogin cuando dice que:

"la reversibilidad de las leyes de la dinámica, lo mismo que las leyes de las dos ciencias fundamentales creadas en el siglo XX - la mecánica cuántica y la teoría de la relatividad - son una expresión radical de una negación del tiempo, que no podria haberse imaginado nunca ninguna cultura, ningún saber colectivo"18

La "Sinergética al proponer otra solución a los problemas que la irreversibilidad del tiempo plantea a la ciencia, coloca en el centro de su análisis la evolución de la estructura de los sistemas autorregulados, buscando en ella los factores que permiten distinguir el pasado y el futuro, y descubrir en ellos la historia, la temporalidad. Este enfoque ha puesto en evidencia, que sobre la llamada "flecha del tiempo" es posible hablar solo cuando existe una coincidencia de la casualidad de los hechos históricos con las incidencias impredecibles del caos. Esta coincidencia es la que genera procesos irreversibles, "hechos contingentes que no conducen necesariamente como única alternativa a la degradación de los sistemas, sino también a niveles superiores de organización. La entropia, por ejemplo, no solo es un estado del sistema que evoluciona hacia su máxima desorganización, hacia el caos, sino que, en determinadas condiciones, puede ser un factor generador de un nuevo orden de un nuevo proceso, de un nuevo sistema.

Esta visión de la entropía tiene un profundo valor metodológico y significa el reconocimiento de que el caos no es una alternativa rígida o un obstáculo para que los sistemas evolucionen hacia una

18

I. Prigoguin. Op. cit. pág. 7. 
mayor complejidad, sino una condición importante para que verdaderamente este desarrollo exista. Se puede decir que con este nuevo enfoque las presuntas diferencias entre el orden físico y el biológico dejan de existir.

El "redescubrimiento" del tiempo implica un cambio radical de nuestra concepción del mundo y, por lo tanto, debe ser objeto de una profunda reflexión filosófica, pues no cabe dudas que el tiempo sigue siendo uno de los temas más importantes de esta forma de la conciencia social

Sería un error si dijéramos que la filosofía de nuestro siglo se ha desentendido de este importante problema, pero no es menos cierto, que ha aportado para su solución mucho menos de lo esperado

Esto lo vio con bastante claridad Bergsón, cuando señaló que ello se debía a la estrecha vinculación de la filosofía con la racionalidad de la ciencia, la que era incapaz de comprender la temporalidad, más allá de la secuencia de los diferentes estados que nos describen las leyes de la mecánica determinista. Pero Bergsón, lo mismo que mas tarde Whitehead, pensaron que la solución debía ser obra exclusiva de la especulación filosófica, desvinculada del racionalismo determinista de la ciencia y consideraron que las alternativas a ese estilo de pensamiento se podían encontrar sólo en la esfera de las formas intuitivas de la aprehensión, que era, según ellos, una de las prerrogativas de la filosofia. Por eso Bergsón, que inicia su "búsqueda" del tiempo en la profundidad de la subjetividad, cuando al final del camino se encuentra con el tiempo físico real, cree que ha fracasado, al no encontrar un puente seguro entre la filosofia y la ciencia. ${ }^{19}$

Al exponer la situación actual del desarrollo de nuestra cultura y los profundos cambios que en ella provocan los nuevos conocimientos y métodos de la ciencia hemos hecho hincapié en el surgimiento de un nuevo cuadro científico del mundo, de un

\footnotetext{
19 Ver H. Bergsón La evolución creadora. En Oeuvres. Paris PUF. 1970. pág. 780. y A. Whitehead. Process and Reality: Essay in Cosmology. N.Y. 1969.
} 
"paradigma global", que ofrece a la reflexión filosófica y en primer término a la filosofía de la ciencia, un amplio repertorio de ideas científicas que implican nuevos principios, enfoques y recursos investigativos que deben ser asimilados para lograr un análisis efectivo y una interpretación correcta del propio proceso del conocimiento teórico, así como un nuevo enfoque de las relaciones de la Sociedad humana y la Naturaleza.

Como se ha señalado, el intento de abordar esta empresa sin renunciar a los esquemas teóricos del pensamiento "tradicional", resultó estrecho y unilateral y no pudo asimilar la enorme complejidad de los nuevos aportes de la ciencia, ni incluir en este análisis importantes factores internos y externos, que hasta el momento no habían sido incorporados a esta empresa. Factores que van desde los ideales, normas y estructuras del saber en general, a las características de los objetos que hoy se encuentra en el centro de la investigación científica: los sistemas complejos de autoorganización.

Todo lo señalado, exige una nueva visión de la estructura y las características del proceso cognoscitivo, sus posibilidades y limitaciones. Pero exige también una metodología y una estrategia científica nueva, así como la elaboración de nuevos métodos de investigación empírica y nuevos procedimientos de descripción de la realidad investigada. Por otra parte, los cambios ya evidentes en las relaciones objeto-sujeto han planteado ante la ciencia y la filosofia la necesidad de elaborar métodos y procedimientos que se adapten a los diferentes niveles de participación del sujeto en la estructura de los procesos investigados. Debiéndose tener presente que según sea este nivel, o si se quiere, el grado de participación del sujeto, el acto investigativo puede, en cierta manera, afectar los resultados de la ciencia.

Ante la necesidad de incorporar todos los factores señalados al análisis filosófico del conocimiento científico se han propuesto diferentes soluciones y buscado nuevos caminos, pues el tradicional, como ya se ha señalado, parece haber agotado todas sus posibilidades y recursos. Se necesita un cambio cualitativo. En este 
sentido nos hemos acordado de la última frase del libro de Suppe, que pertenece a un "Postscriptum" de S.Taulmin y que dice así:

"Cuando la filosofia de la ciencia alcance este estadio, sin embargo, no tendrá ya ninguna relación con la "imagen tradicional" de las teorías científicas o "calculo axiomático". Mas bien una tradición completamente nueva habrá nacido" 20

$\mathrm{Si}$ esa tradición ha nacido ya o no, aún es difícil opinar, pensamos que se está gestando y que los elementos básicos para su elaboración ya se pueden enumerar y hasta clasificar. Creemos que, en parte, hemos intentado señalar algunas pistas y expuesto algunas ideas de como se intenta lograr, pero seguirlas en detalle es un tema especial, que rebasa los marcos de nuestra exposición. Y una observación más.

En la literatura filosófica y la historia de la ciencia durante los últimos decenios se ha trabajado en la elaboración de una idea más amplia que la del concepto de teoría científica, en los marcos de la cual fuera posible realizar esa síntesis y esa fundamentación del saber científico contemporáneo y su historia, que con tanta urgencia se está buscando. Nos referimos al concepto de "programa científico" introducido al parecer por I. Lakatos en $1971^{21}$ en el que quedarían incluidas no solo las diferentes teorías científicas, sino también todas aquellas ideas, postulados, principios y afirmaciones que se aceptan por las diferentes ciencias, pero que no se demuestra ni fundamentan dentro de estas.

Las causa que llevaron a la formulación de este concepto son evidentes y van, desde la imposibilidad de explicar los cambios radicales que tienen lugar en las ciencias naturales, hasta la

\footnotetext{
20 F. Suppe. Op. cit. pág. 492.

21

I. Lakatos "History of Science and its Rational Reconsuructions." in Buck and Cohen 1975. Ver, Piama Gaidenko. La evolución del concepto ciencia. La génesis y el desarrollo de los primeros programas científicos. Moscú, 1980, 567 págs. La evolución del concepto ciencia (siglos XVII-XVII). La formación de los programas científicos de la época moderna. Moscú 1987.447 págs.
} 
necesidad de encontrar los principios que garantizan la unidad teórico-metodológica del saber en general.

A diferencia de las teorias científicas, cuyo alcance es relativamente limitado, el "programa científico tiene un carácter más general $y$, hasta pudiera decirse que el principio o sistema de principios que lo conforman tiene pretensiones "universales", al intentar una explicación coherente de toda la realidad. Por ejemplo, el principio de los pitagóricos "todo es un numero" es un ejemplo de una formulación lacónica de un programa científico. Con la elaboración de este concepto se está intentando abrir un camino que permita descubrir y exponer la evolución del pensamiento científico sin perder de vista su carácter específico y relativa independencia, pero sin absolutizar estos factores, sin alterar la vinculación orgánica de la ciencia con los diversos componentes de la cultura y entre ellos, en primer lugar, la filosofía. 